\title{
Sub-Ångström-resolution MicroED Using a Direct Detection Camera
}

\author{
Benjamin Bammes ${ }^{1}$, Weijiang Zhou ${ }^{2}$ and Wah Chiu ${ }^{2}$ \\ ${ }^{1}$ Direct Electron, LP, San Diego, California, United States, ${ }^{2}$ Stanford University, Menlo Park, California, \\ United States
}

The recent development of microcrystal electron diffraction (MicroED) in electron cryo-microscopy (cryo-EM) has addressed many of the challenges of X-ray crystallography [1], filling a long-standing need in organic chemistry for rapid and accurate high-resolution structural characterization [2]. Like X-ray crystallography, this is also a diffraction technique requiring crystalline specimens, but because high energy electrons have a more favorable ration of elastic to inelastic scattering [3], MicroED can acquire data using crystals that are orders of magnitude smaller than those needed for X-ray crystallographyeven as small as $50 \mathrm{~nm}$ in length [4]. Additionally, the instrumentation required for MicroED is readily available, as many chemistry and biology labs already have access to cryo-capable transmission electron microscopes (TEM).

One of the critical hinderances to the broad adoption of this technique is its current requirement for a specialized TEM camera system [5]. Extending this technique to use the same direct detection camera systems already present in cryo-EM labs would significantly eliminate a large (and costly) barrier to adoption of this technique and allow it to reach its full potential for propelling widespread scientific progress. Additionally, the improved capabilities of direct detection cameras [6,7] compared to the scintillator-based cameras currently used for MicroED have the potential to significantly improve data quality, provided that they also have sufficient dynamic range to capture diffraction patterns. Direct detection cameras can deliver a much higher framerate, significantly improved single-electron signal-tonoise ratio, and significantly higher resolution than scintillator-based cameras.

We acquired continuous-rotation MicroED data using a DE-64 direct detection camera (Direct Electron LP, San Diego, CA) mounted on a Talos Arctica $200 \mathrm{keV}$ TEM (Thermo Fisher, Waltham, MA). Small molecule biotin (Sigma-Aldrich, St Louis, MO) was used as a test sample in our experiment. This lyophilized powder was directly extracted from the commercial bottle, ground between two glass slides, and placed on a glow-discharged Quantifoil R1.2/1.3 Cu300 grid. The grid was then vitrified by manually plunging into liquid nitrogen.

Nano-sized crystals were searched using low-dose high-defocus diffraction mode to minimize radiation damage. For each crystal found, a low-dose trial shot (1 s exposure) was acquired with in-focus diffraction mode to examine whether the crystal diffracted to high-resolution. If so, a custom SerialEM [8] script was executed to record a continuous stream of frames on the DE-64 using in-focus diffraction patterns while the specimen stage was continuously rotated from $-50^{\circ}$ to $+50^{\circ}$ at $0.9^{\circ} \mathrm{s}$. The frame rate of the DE- 64 was set to 20 frames per second (fps) and the total exposure time to 120 seconds. The exposure rate was 0.03 $\mathrm{e}^{-} / \AA^{2} / \mathrm{s}$, yielding a total accumulated dose of about $3.6 \mathrm{e}^{-} / \AA^{2}$. Indexing and integration was completed using XDS [9], followed by ab initio structure determination and structure refinement using SHELXT and SHELXL [10].

From a single acquisition of one biotin microcrystal, we obtained a $0.7 \AA$ A-resolution map (Fig. 1) with exceptional goodness of fit (Fig. 2). All four types of atoms (C, O, N, S) were assigned correctly and hydrogen atoms were clearly positioned in the refined model as well. These results demonstrate that the 
DE-64 direct detection camera may be used to rapidly generate atomic resolution models using MicroED [11].

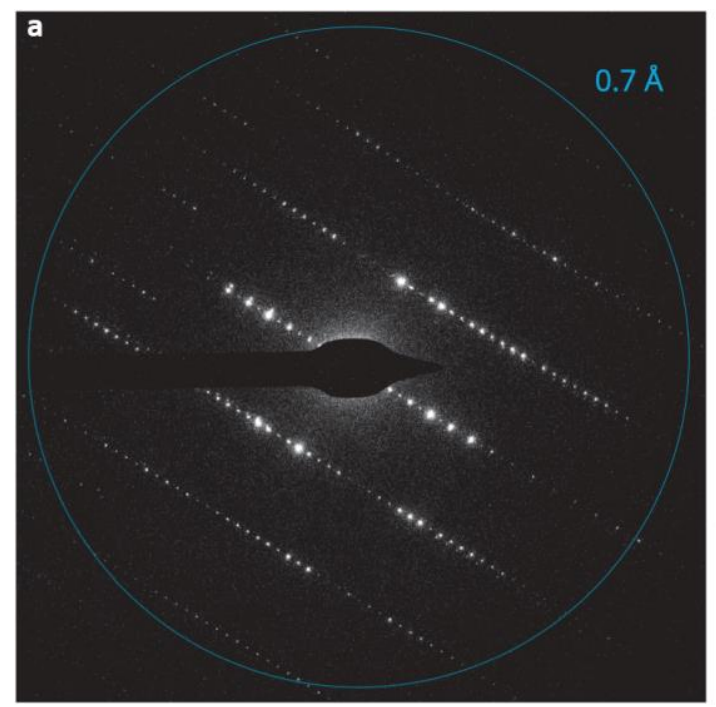

b

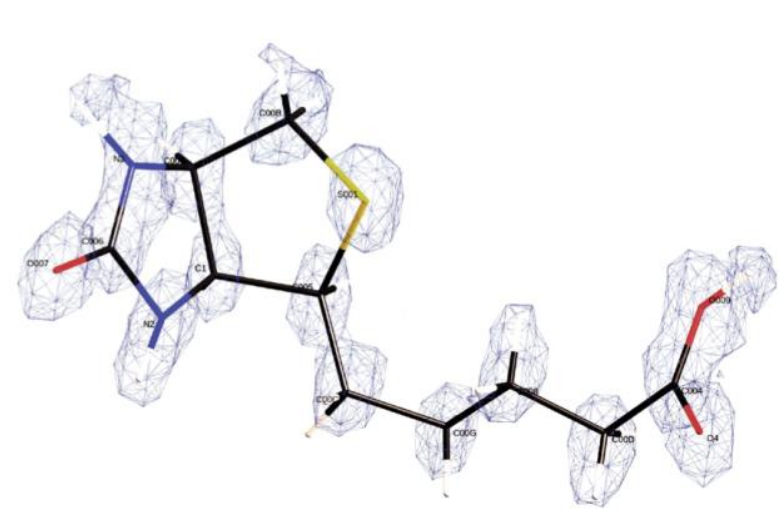

Figure 1. (a) A sum of 60 frames, representing about $3^{\circ}$ of total rotation, from the movie collected on the DE-64 direct detection camera during continuous-rotation MicroED. Diffraction spots are clearly visible out to approximately $7 \AA$ resolution. (b) The resulting density map and atomic model after processing using XDS and SHELX.

\begin{tabular}{|l|l|}
\hline Temperature $(\mathrm{K})$ & 100 \\
\hline Unit cell lengths $(\AA)$ & $5.440(2), 10.640(2), 21.710(4)$ \\
\hline Angles $\left(^{\circ}\right)$ & $90.0(3), 90.0(3), 90.0(3)$ \\
\hline Space group & $\mathrm{P} 2_{1} 2_{1} 2_{1}$ \\
\hline Reflections & $5031(526)$ \\
\hline Unique reflections & $1439(162)$ \\
\hline $\mathrm{R}_{\text {obs }}$ & $21.5(31.5)$ \\
\hline $\mathrm{R}_{\text {meas }}$ & $25.1(37.1)$ \\
\hline CC $1 / 2$ & $96.5(82.0)$ \\
\hline Resolution $(\AA)$ & 0.7 \\
\hline Completeness $(\%)$ & $79.3(86.6)$ \\
\hline Exposure $\left(\mathrm{e}-/ \AA \AA^{2}\right)$ & $\sim 3.6$ \\
\hline$R$ & 0.211 \\
\hline GooF & 1.052 \\
\hline
\end{tabular}

Figure 2. The final statistics for the model generated by our MicroED data set of biotin.

\section{References}

[1] Shi D., Nannenga B.L., Iadanza M.G., Gonen T. (2013). Elife, 2: e01345.

[2] Jones C.G., Martynowycz M.W., Hattne J., Fulton T.J., Stoltz B.M., Rodriguez J.A., Nelson H.M., \& Gonen T. (2018). The CryoEM method MicroED as a powerful tool for small molecule structure determination. ACS Central Science, 4: 1587-1592. 
[3] Henderson, R. (1995). The potential and limitations of neutrons, electrons and X-rays for atomic resolution microscopy of unstained biological molecules. Quarterly Reviews of Biophysics, 28: 171-193. [4] Rodriguez J.A., Ivanova M.I., Sawaya M.R., Cascio D., Reyes F.E., Shi D., Sangwan S., Guenther E.L., Johnson L. M., Zhang M., Jiang L., Arbing M.A., Nannenga B. L., Hattne J., Whitelegge J., Brewster A.S., Messerschmidt M., Boutet S., Sauter N.K., Gonen T., \& Eisenberg D. S. (2015). Nature, 525: 486490.

[5] Hattne J., Martynowycz M.W., Penczek P.A., \& Gonen T. (2019). MicroED with the Falcon III direct electron detector. IUCrJ, 6: 921-926.

[6] Bammes B.E., Rochat R.H., Jakana J., Chen D-H., \& Chiu W. (2012). Direct electron detection yields cryo-EM reconstructions at resolutions beyond 3/4 Nyquist. Journal of Structural Biology, 177: 589-601.

[7] Faruqi A.R. \& McMullan G. (2011). Electronic detectors for electron microscopy. Quarterly Reviews of Biophysics, 44: 357-390.

[8] Mastronarde D.N. (2005). Automated electron microscope tomography using robust prediction of specimen movements. Journal of Structural Biology, 152: 36-51.

[9] Kabsch W. (2010). XDS. Acta Crystallographica Section D, 66: 125-132.

[10] Sheldrick G.M. (2008). A short history of SHELX. Acta Crystallographica Section A, 64: 112-122.

[11] This material is based upon work supported by the U.S. Department of Energy, Office of Science, under Award Number DE-SC0020550, and by the U.S. National Institutes of Health, National Institute of General Medical Sciences, under Award Numbers 5P41GM103832 and S10OD021600. 